\title{
Probable Vertical Transmission of SARS-CoV-2 Infection in the Countryside of São Paulo State, Brazil: Case Report
}

\author{
(1) Patricia Rodrigues Naufal Spir1, (1) Breno Miranda Souza1', (1) Verena Miranda Souza1, \\ (1) Julia Arruda Costa Galvão2, (1) Luisa Arruda Da Costa Galvão3, (1) Luiz Euribel Prestes-Carneiro4 \\ 1Oeste Paulista University, Presidente Prudente, Department of Pediatrics, São Paulo, Brazil \\ 2Universidade of Taubaté Faculty of Medicine, Department of General Medicine, São Paulo, Brazil \\ 3/piranga Hospital, Department of Ginecology and Obstetrics, São Paulo, Brazil \\ ${ }^{4}$ Oeste Paulista University, Presidente Prudente, Department of Public Health, São Paulo, Brazil
}

\begin{abstract}
We reported a probable vertical transmission of severe acute respiratory syndrome-Coronavirus-2 (SARS-CoV-2) infection in Presidente Prudente, a city of the western region of São Paulo. Furthermore, regionally updated data on SARS-Coronavirus diseases-2019 pregnant women pinpointed the vulnerability of the region with higher levels of infected and dead mothers compared to nationwide data.
\end{abstract}

Keywords: SARS-CoV-2, vertical transmission, pregnant women

\section{Introduction}

Worldwide, Brazil ranks third for the number of individuals infected with severe acute respiratory syndromeCoronavirus-2 (SARS-CoV-2) and second with regard to deaths. São Paulo, the richest and most populous state, is the epicenter and ranks first for the number of infected patients and deaths. Considered one of the poorest and most undeveloped regions, western São Paulo comprises 45 municipalities with an estimated population of 753,344 inhabitants. In May 2020, pregnant and postpartum women were included in the group most susceptible to the effects of Coronavirus diseases-2019 (COVID-19) in Brazil. Transient immunosuppression for maintaining the tolerance of fetal semi allograft makes them more vulnerable to viral infections. Furthermore, changes in the respiratory and circulatory systems predispose pregnant women to worse clinical outcomes when infected with viruses such as the H1N1 virus (1). In December 2020, the nationwide profile of pregnant women with SARS who were hospitalized and deaths confirmed by COVID-19 were updated. From February to December 2020, 4,467 of 9,581 (44.7\%) pregnant women were diagnosed with COVID-19 and 233 (5.2\%) died (2). However, although mothers and their newborns should be considered a high-risk population in the development of prevention and management strategies for COVID19, in Brazil, little public data are available on vertical transmission, and cases are restricted to case reports (3-7). 


\section{Case Report}

A 35-year-old gravida 2, para 1 was admitted to a private hospital in Presidente Prudente, in the countryside of São Paulo, Brazil, on July 15, 2020, at 35+5 weeks of gestation, and up to that time, the pregnancy had been uneventful. She was referred with rhinorrhea and body pain and presented premature labor with no cause and clear amniotic liquid. She had controlled insulin-dependent type I diabetes mellitus and was reported to have been in contact with her sister who had been diagnosed with COVID-19. Despite epidemiological data, the obstetrician and the patient did not consider the possibility of COVID-19 infection and the viral load of the amniotic fluid and umbilical cord were not collected. She had had no other symptoms and was not treated for COVID-19. A cesarean delivery was performed with rupture of the amniotic membranes, with clear amniotic liquid, without lumps or meconium.

A male neonate was delivered; the birth weight was 3,100 g, Apgar scores were 9 and 10 at 1 and 5 min, respectively. Gestational age according to the New Ballard score was 35 weeks and 5 days. He was taken to a bath in running water immediately after delivery, without contact with the mother after birth. Still in the delivery room, he developed tachypnea and moaning (Silverman Andersen Bulletin=6), and was taken to the neonatal intensive care unit (NICU). He was placed in an incubator with fasting, probe open orogastric tubes, maintenance serum, and continuous positive airway pressure with an inspired oxygen fraction $\left(\mathrm{FIO}_{2}\right)$ of $30 \%$ and aerosol precautions. Chest radiography was performed, showing alterations compatible with pulmonary interstitial edema. His initial diagnoses were preterm newborn, suitable for age pregnancy, premature labor, and early respiratory distress. A real-time reverse transcriptase-polymerase chain reaction (rRT-PCR) test for nasopharyngeal SARS-CoV-2 RNA was performed with indeterminate results on day 2 (July 17, 2020) and positive results on day 7 (July 21, 2020). Nasopharyngeal samples were collected from the mother for COVID-19-rRT-PCR and the result was positive. The newborn remained in the NICU until day 7, under specific precautions. No contact with his mother or family, and no breastfeeding were allowed. On day 9, chest tomography was performed, showing consolidation in the upper segment of the left lower lobe associated with discrete bilateral ground-glass opacities (Figure 1). Laboratory values were at normal levels on day 1 and in the following segment. The newborn showed progressive improvement and was discharged on day 11 weighing 2,940 g, and anti-COVID-19 IgG and total antibodies were positive. Written informed consent was

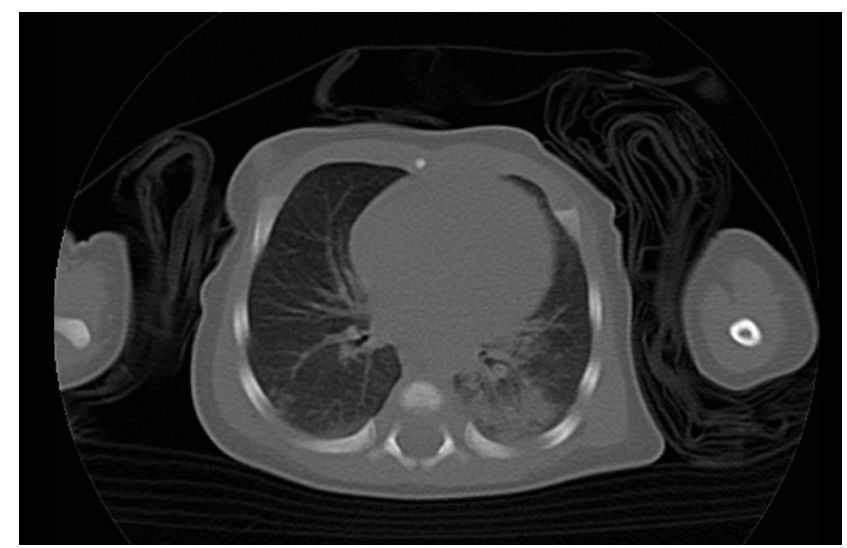

Figure 1. Chest tomography: Axial section with window for viewing parenchyma performed on the $9^{\text {th }}$ day of hospitalization

obtained to publish this paper. The project was approved by the Ethical Committee of Oeste Paulista University, Brazil. Protocol number 4.469.118; December 16, 2020.

\section{Discussion}

It is possible to consider vertical transmission of COVID19 in the newborn in this case. According to the criteria of the São Paulo Pediatric Society, suspected and confirmed cases in pregnant women and newborns are defined when the newborn has a positive result for COVID-19 by RT-PCR in respiratory tract samples with swab collection. (https:// www.spsp.org.br/2020/03/30/coronavirus-e-recemnascido-o-que-se-sabe-ate-o-momento-30-03-2020/). Following the delivery, strict measures were taken to reduce the risk of in fection, and the newborn did not have contact with his mother or other family members, a fact that strongly supports the diagnosis. Although congenital SARS-CoV-2 infection can be confirmed by PCR of placental tissue and the umbilical cord, few reports are available using this methodology in Brazil (4), and in most cases, the results were obtained from nasal/oropharynx swabs. In São Paulo, in 3 cases reports of vertical transmission of COVID-19, positive results for SARS-CoV-2 by RT-PCR were obtained from oropharynx swabs (5). In São Luis, Maranhão state, a positive result in RT-PCR4 for SARSCOV-2 was obtained from nasal swab at 6 hours after birth and a probable vertical transmission was described (6). In Rio de Janeiro, nasal and oropharynx swabs were also collected, and RT-PCR tests for SARS-CoV-2 were positive in a maternal SARS-CoV-2 infection associated with systemic inflammatory response and pericardial effusion in the newborn (7). Symptoms of groaning and tachypnea started immediately after birth and the newborn was sent to the NICU for ventilatory support. Chest tomography 
demonstrated alterations compatible with pulmonary interstitial edema and consolidation associated with ground-glass opacities. Respiratory manifestations are among the main clinical symptoms reported in newborns infected with COVID-19, and about 30\% require admission to the NICU. Furthermore, chest imaging examination should be actively performed in newborns suspected of COVID-19 infection (8). In a review of newborns infected with COVID-19, non-specific radiographic findings were described in $32 \%$ of cases, with ground-glass opacity in $8 \%$ and linear opacities in 4\% (9).

The delivery and neonatal support in this case occurred in a tertiary private hospital with an available NICU in the countryside of São Paulo state. However, such facilities are not available in most regions of Brazil, including the municipalities of western São Paulo, which only has 5 NICUS, all of them being in the city of Presidente Prudente. In 2020, in the western region, 29 pregnant women were diagnosed with SARS, and 15 (51.7\%) were diagnosed with COVID-19; two deaths occurred (13.3\%), and one infected newborn died. These rates are higher than the countrywide rates, not only for infections ( $51.7 \%$ vs $44.7 \%$ ), but also for deaths of pregnant women (13.3\% vs $5.2 \%)$. All the cases of vertical transmission of COVID-19 occurred in large cities in different regions of Brazil, including São Paulo and Rio de Janeiro in the southeast, Curitiba in the south, and São Luis in the northeast (3-7).

\section{Conclusion}

This case report focuses on a newborn in a city of São Paulo State. Furthermore, regionally updated data on pregnant women with SARS-COVID-19 demonstrates the asymmetry and vulnerability of regions with higher levels of infected mothers and deaths compared with the nationwide data.

\section{Ethics}

Informed Consent: Written informed consent has been obtained to publish this paper.

Peer-review: Externally peer-reviewed.

\section{Authorship Contributions}

Medical Practices: P.R.N.S., Concept: P.R.N.S., L.E.P.C., Design: P.R.N.S., Data Collection or Processing: B.M.S., J.A.C.G., V.M.S., L.A.C.G., Analysis or Interpretation: P.R.N.S., L.E.P.C., Literature Search: P.R.N.S., B.M.S., I.A.C.G., V.M.S., L.A.C.G., L.E.P.C., Writing: L.E.P.C., P.R.N.S.

Conflict of Interest: No conflict of interest was declared by the authors.

Financial Disclosure: The authors declared that this study received no financial support.

\section{References}

1. Cornish EF, Filipovic I, Åsenius F, Williams DJ, McDonnell T. Innate Immune Responses to Acute Viral Infection During Pregnancy. Front Immunol 2020 30; 11:572567.

2. Ministério da Saúde Secretaria de Vigilância em Saúde. Doença pelo Coronavírus COVID-19. Boletim Epidemiológico Especial 2020: 45.

3. Rebello CM, Fascina LP, Annicchino G, Pinho JRR, Yoshida RAM, Zacharias RSB. Vertical transmission of SARS-CoV-2 from infected pregnant mother to the neonate detected by cord blood real-time polymerase chain reaction (RT-PCR). Pediatr Res 2020 Oct 26.

4. Stonoga ETS, de Almeida Lanzoni L, Rebutini PZ, et al. Intrauterine Transmission of SARS-CoV-2. Emerg Infect Dis 2021; 27:638-41.

5. De Bernardo G, Giordano M, Zollo G, et al. The clinical course of SARS-CoV-2 positive neonates. I Perinatol 2020; 40:1462-9.

6. Pessoa FS, Vale MSD, Marques PF, Figueira SDS, Salgado IADSC, Mochel RSWC. Probable vertical transmission identified within six hours of life. Rev Assoc Med Bras 2020; 66:1621-4.

7. Lima ARO, Cardoso CC, Bentim PRB, et al. Maternal SARS-CoV-2 infection associated to systemic inflammatory response and pericardial effusion in the newborn: a Case-Report. J Pediatric Infect Dis Soc 2021; 10:536-9.

8. Li HY, Zhang CM, Lv YY, Dai WQ, Xu B, Qi XM. Value of chest imaging in the newborn with suspected COVID-19. Eur Rev Med Pharmacol Sci 2020; 24:11971-6.

9. De Bernardo G, Giordano M, Zollo G, et al. The clinical course of SARS-CoV-2 positive neonates. J Perinatol 2020; 40:1462-9. 